\title{
Digital twin development of a solar power plant
}

\author{
Ludmila Massel $^{1}$, Nikita Shchukin ${ }^{1}$, and Alexey Cybikov ${ }^{1}$ \\ ${ }^{1}$ Melentiev Energy Systems Institute, 130 Lermontov str., Irkutsk, Russia
}

\begin{abstract}
The article is devoted to the development of a digital twin of a solar power plant. An overview of existing solutions in this area is given. An approach to building a digital twin based on ontological engineering is discussed in more detail. The main steps of ontological engineering are described. A fragment of the system of ontologies of photovoltaic systems is presented. A mathematical model designed to simulate a solar power plant taking into account changes in environmental parameters is considered. The article also focuses on the design of the architecture of the digital twin, consisting of: Digital Shadow, Digital Model and control system. The article also describes the stage of implementation of a prototype of a digital twin of a solar power plant, which currently includes a database and a component for calculating the output characteristics of solar panels and inverters.
\end{abstract}

\section{Introduction}

The creation of digital twins is one of the trends in the development of energy technologies and in the energy digitalization. Digitalization is currently one of the modern direction of energy development [1].

Consumers have access to centralized power supply not at all the entire territory of the Russian Federation. As a result, it is necessary to bring fuel to remote settlements, which is used for the operation of diesel power plants. This is an unprofitable and not a permanent source of energy.

For these settlements, it is advisable to create solar power plants that increase the availability of electricity, but their construction is a large one-time investment and requires high-quality maintenance of already built facilities.

Therefore, it makes sense to simulate them in advance, where digital twin technology can help.

A digital twin is a virtual prototype of a real object, with which you can conduct experiments and test hypotheses, predict the behavior of an object and solve the problem of managing its life cycle. The digital twin also saves equipment or system design and operating costs. So, for example, when using a digital twin, it becomes possible to determine the actual time for carrying out work on the replacement or repair of equipment.

We can conclude that the use of digital twins in the design of solar power plants can have a positive effect both at the design and development stage, and at the stage of their operation.

\section{Digital twins}

Within the framework of the Industry 4.0, an important element of an intelligent and high-tech control system is the concept of digital twins [2]. Currently, digital twins have proven themselves well in many areas such as oil and gas industry, engineering, urban planning, etc. $[3,4,5,6]$. The introduction of digital twins into energy facilities increases the possibilities in the design, forecasting and management of these systems by means of structural modeling of objects and production processes, as well as their imitation. activities using the developed model.

According to the sources [7-10], it can be concluded that today there is no strict definition of the digital twin concept, however, the general meaning of such a term is to create a constantly updated digital model used for the execution of experiments and testing hypotheses, forecasting behavior of an object and solving the problem of managing its life cycle, based on real or experimental data $[11,12]$. The main purpose of developing a digital twin is to transfer an object to a digital space, to simulate a change in the state of an object under the influence of various factors and possible control actions, to determine and implement optimal control actions to achieve the target state of the object.

The analysis of research carried out in the field of using the concept of digital twins $[10,13]$ confirms the relevance of their construction for solar power facilities.

The authors propose to use an ontological approach based on ontological engineering for the development of digital twins.

\section{Ontological engineering}

The goal of ontological engineering is to structure and manage knowledge using ontologies. For a better understanding of this term, we use a detailed definition of ontology in computer science [14]. Ontology is a formal specification of a shared conceptual model where

- a "conceptual" model means an abstract model of the subject area that describes the system of concepts in this area;

- "shared" means the agreed understanding of the conceptual model by a certain community (group of people);

- "specification" means an explicit description of a system of concepts; 
- "formal" means that the conceptual model is given in a formalized language.

It should be noted that the visual component of ontologies is a powerful tool in the process of structuring and presenting knowledge. For the visual design of ontologies, a four-step algorithm was used [15] described below:

Step 1. Goals, strategy and boundary identification: - Determination of the purpose of the ontology and the needs for acquiring knowledge in the subject area.

As a result of this stage, the goal was set to build a system of ontologies of a solar power plant and integrate it into the existing ontology of the fuel and energy complex, as well as acquire knowledge in the field of solar power engineering to build a digital twin.

- Determine the scope or "boundaries" of the ontology and the type of ontology before proceeding to step 2 .

The developed ontology is of the heuristic type and will be used to build a digital twin.

Step 2. Glossary development or meta-concept identification: It is necessary to collect all information related to the described area. The main goal of this step is to select and verbalize all the main objects and concepts in the field. The result of the step is shown below:

- Generation system;

- Renewable energy sources;

- Photovoltaic systems (PS);

- PS equipment;

- Illumination.

Step 3. Laddering, including categorization and specification: The next step is to define all the main objects and concepts of the domain (main levels of abstraction). Identifying a structured hierarchy is one of the main goals at this stage. The hierarchy built using the results of the previous step is shown below:

* electric power systems;

$>$ generation systems;

- renewable energy sources;

- photovoltaic systems (PS);

- PS equipment;

- illumination.

Step 4. Refinement: The last step is dedicated to updating the visual structure by eliminating any excess, synonymy and contradiction.

In this step, the concepts and the relationships between them were reworked, which led to an improvement in the comprehensibility and readability of the ontology system.

As a result of the execution of the four-stage algorithm, a system of ontologies of a solar power plant was constructed, presented in the figures below:

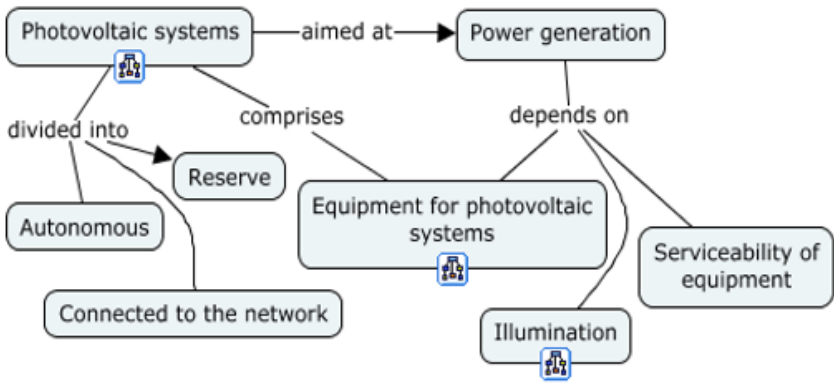

Fig. 1. Photovoltaic systems

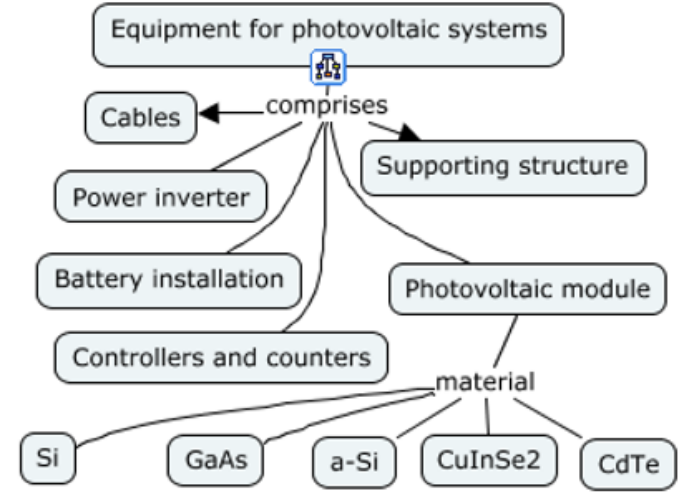

Fig. 2. Equipment for photovoltaic systems

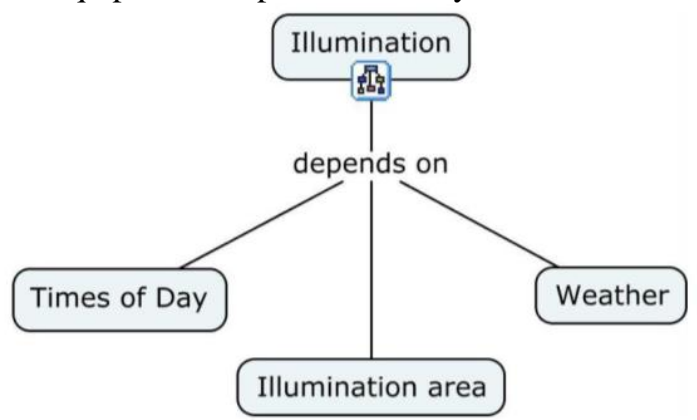

Fig. 3. Illumination

\section{Mathematical model}

To model a solar power plant taking into account changes in environmental parameters, the approach proposed by D.N. Karamov. and Naumov I.V. is used [16].

To calculate the generated power, efficiency and temperature of the solar panel, as well as the output voltage and current, you need to use the following parameters: solar radiation, air temperature, wind speed and panel characteristics.

The formula (1) calculates the level of output power on the photoelectric converter.

$$
P_{p v}(t)=\bar{I}_{A}(t) \eta(t) A k_{L},
$$

where $\bar{I}_{A}(t)$ is the solar radiation arriving at the inclined surface of the photovoltaic converter, $\mathrm{W} / \mathrm{m}^{2} ; \eta(t)$ is the efficiency of the photoelectric converter, rel. units; A is the total installed area of solar panels, $\mathrm{m}^{2} ; k_{L}$ - coefficient taking into account losses in diodes, is taken equal to 0.85 0.95 rel. units

The calculation of the efficiency takes place according to the formula (2).

$$
\eta(t)=\bar{\eta}\left[1-\beta\left(T_{p v}(t)-48\right)\right],
$$

where $\bar{\eta}$ is the passport value of the efficiency of the photoelectric converter, rel. units; $\beta$ is the temperature coefficient for silicon photovoltaic converters, rel. units $/{ }^{\circ} \mathrm{C}$.

To determine the temperature on the working surface of the photoelectric converter $\left[T_{p v}(t)\right]$, it is required to use the Koehl correlation coefficients $\left(k_{0}, k_{1}\right)$ and the wind speed on the earth's surface $\left[V_{w}(t)\right]$ in formula (3).

$$
T_{p v}(t)=T_{a}(t)+\frac{\bar{I}_{A}(t)}{k_{0}+k_{1} V_{w}(t)},
$$

Next, we calculate the photo stream: 


$$
I_{P H}(t)=\frac{\bar{I}_{A}(t)}{1000}\left[I_{S C}+k_{I}\left(T_{p v}(t)-25\right)\right],
$$

where $I_{S C}$ is the short-circuit current.

After calculating the output power and photocurrent of photovoltaic converters, it became permissible to calculate the output voltage:

$$
U_{p v}(t)=\frac{P_{p v}(t)}{I_{P H}(t)} .
$$

The presented mathematical model determines the necessary parameters and has advantages that are associated with the change in weather conditions and the interaction of the elements of the photoelectric converter.

The considered model is necessary to obtain data on the behavior of the system. The use of this data makes it possible for the digital twin to predict the behavior of an object and solve the problem of managing its life cycle.

\section{Database design and construction}

When designing and operating a digital twin of a solar power plant, it is necessary to use a large amount of data, namely: characteristics of weather conditions, characteristics of equipment, etc.
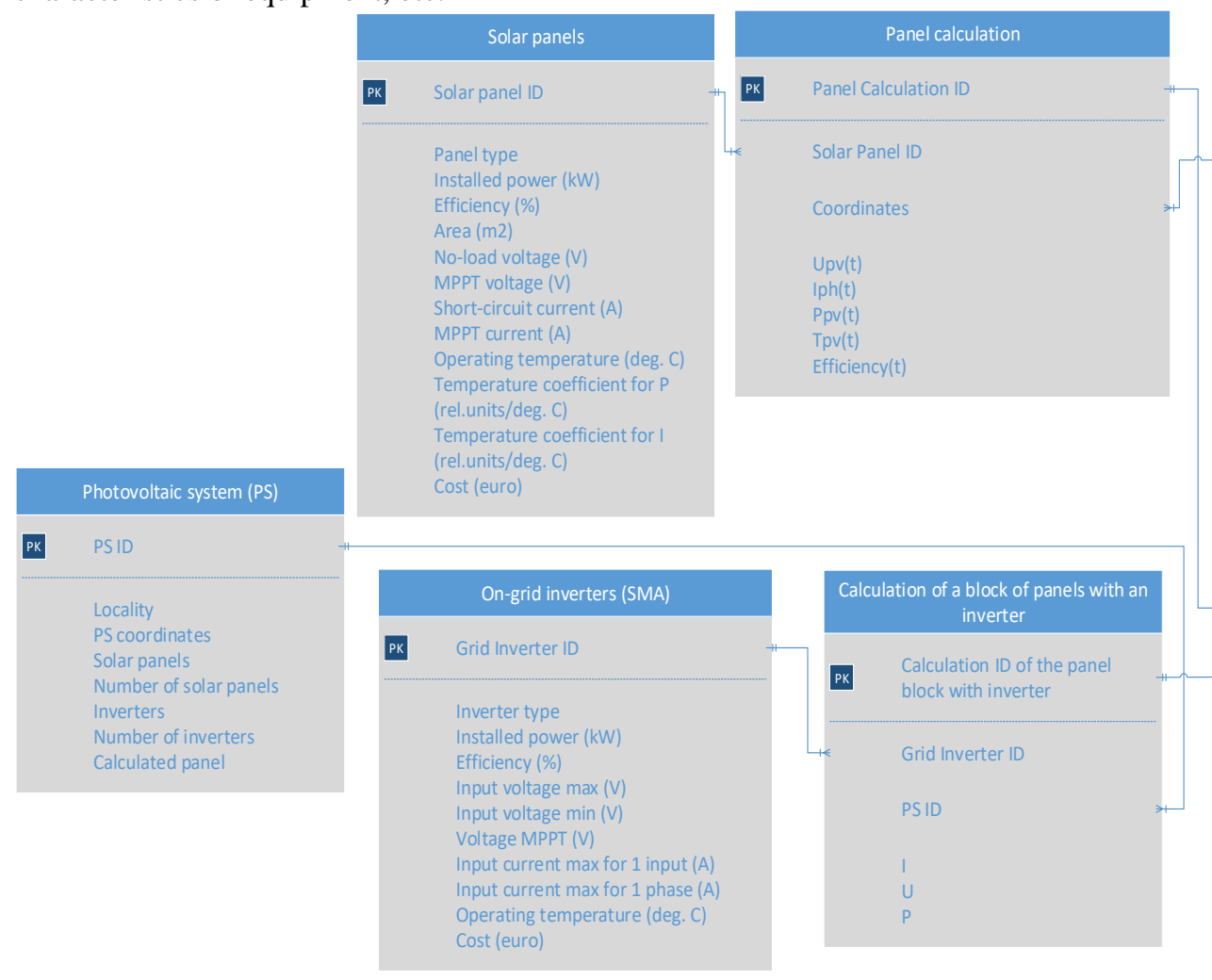

This dataset needs to be organized and stored. For this, it was decided to use a relational database. The PostgreSQL DBMS was chosen because it meets all the requirements listed below.

1) Functionality and convenience:

a) developed SQL language, ease of development and compactness of the code;

b) the ability to run on a variety of OS: Windows, Linux, NIX, BSD, MacOS;

c) ease of administration.

2) Resource intensity.

The design of the database was carried out on the basis of ontologies built in the section "ontological engineering". As a result of the design, the following entities were identified:

- weather characteristics;

- solar panels;

- network inverters (SMA);

- panel calculation;

- calculation of a block of panels;

- calculation of a block of panels with an inverter;

- photovoltaic system.

Relationships between entities are shown in Figure 4 below.

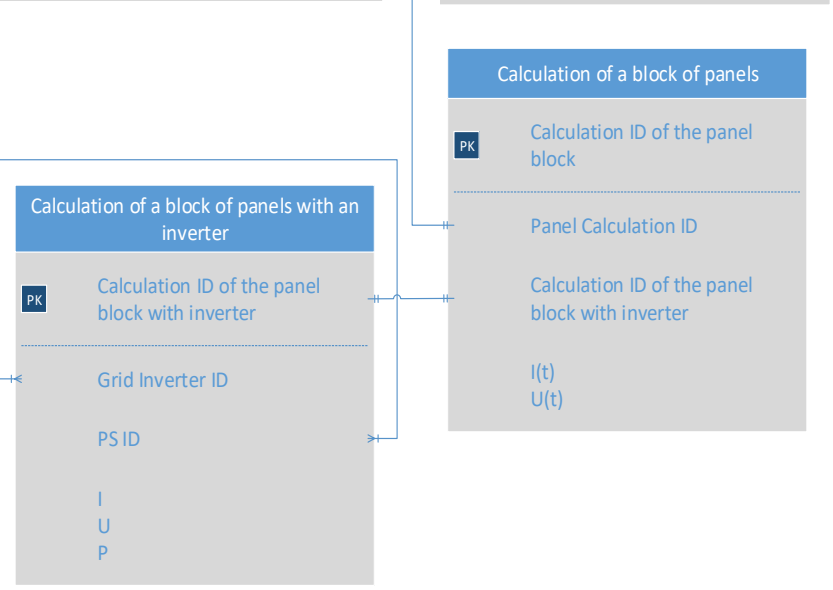

Fig. 4. Database entities and connections between the 


\section{Database interface}

It is possible to work with the database using the administration tools provided by the PostgreSQL DBMS. The editing interface is shown below:

\begin{tabular}{|r|l|l|l|}
\hline & $\begin{array}{l}\text { locality } \\
\text { character varying (15) }\end{array}$ & $\begin{array}{l}\text { hour_number } \\
\text { integer }\end{array}$ & $\begin{array}{l}\text { wind_speed } \\
\text { smallint }\end{array}$ \\
\hline 84 & Baikalsk & 105037 & 1 \\
\hline 85 & Baikalsk & 105036 & 1 \\
\hline 86 & Baikalsk & 105035 & 2 \\
\hline 87 & Baikalsk & 105034 & 3 \\
\hline
\end{tabular}

Fig. 5. PostgreSQL interface

To add and delete records in a table, it is necessary to use SQL queries, which, in turn, complicates the work with the Digital Twin, so it was decided to develop its own interface for working with the database, including the following forms:

- forms for editing (adding / deleting / changing) tables;

- form providing the ability to connect solar panels to inverters under special conditions (the number of ports in the inverter, connecting only the same panels to the inverter, etc.).
An example of the developed interface is shown in Figure 6 at the bottom of the page.

\section{Digital twin architecture}

The digital twin architecture is shown in Figure 7 at the bottom of the page.

The architecture includes the following blocks:

- Digital shadow:

Includes a database and interfaces for working with it, which are responsible for interacting with the database and implement the possibility for the functioning of the used machine learning methods, which are necessary to restore the system behavior model, as well as to predict subsequent data used for the digital twin to work;

- Digital model:

It is necessary for the operability of the entire system as it implements the application interface used for convenient interaction with all other components of the digital model, such as a mathematical model and a system for collecting operational information;

- Control system:

It is used to introduce a control action on a real object, the prototype of which is a digital twin.

\begin{tabular}{|c|c|c|c|c|c|c|c|}
\hline \multicolumn{8}{|l|}{$\begin{array}{l}\text { D Nameran } \\
\text { Фaйn }\end{array}$} \\
\hline Назезние панели & Плочцань С....(M^2) & KRA (\$) & $\operatorname{Tok} \times 3(A)$ & Teun, ко3ф. Ana P & Tesn. кos\$. Ans 1 & \multicolumn{2}{|l|}{ Hasearve novers } \\
\hline Panel1 & 1.63 & 15.6 & 8.45 & 0.005 & 0.00038 & \multirow{2}{*}{\multicolumn{2}{|c|}{ 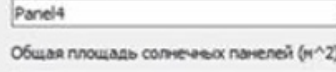 }} \\
\hline Panel2 & 1.63 & 17.2 & 9.31 & 0.0045 & 0.00038 & & \\
\hline \multirow[t]{8}{*}{ Panel3 } & 1.55 & 13.2 & 7.31 & 0.0056 & 0.00038 & \multicolumn{2}{|c|}{$2,52=$} \\
\hline & & & & & & \multicolumn{2}{|c|}{ nochoptroe roverve KIA (\%) } \\
\hline & & & & & & \multicolumn{2}{|l|}{$\operatorname{Tax} K 3(A)$} \\
\hline & & & & & & 8,31 & $:$ \\
\hline & & & & & & \multicolumn{2}{|c|}{ 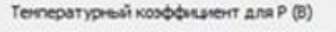 } \\
\hline & & & & & & 0,0065 & $:$ \\
\hline & & & & & & \multicolumn{2}{|c|}{ 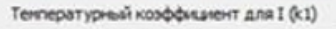 } \\
\hline & & & & & & 0,00038 & $:$ \\
\hline \multicolumn{2}{|c|}{ Ao6sempro } & & nestro & \multicolumn{2}{|r|}{ YAaMTb } & & \\
\hline & & & & & & Omeserts & Bobouto \\
\hline
\end{tabular}

Fig. 6. Program interface

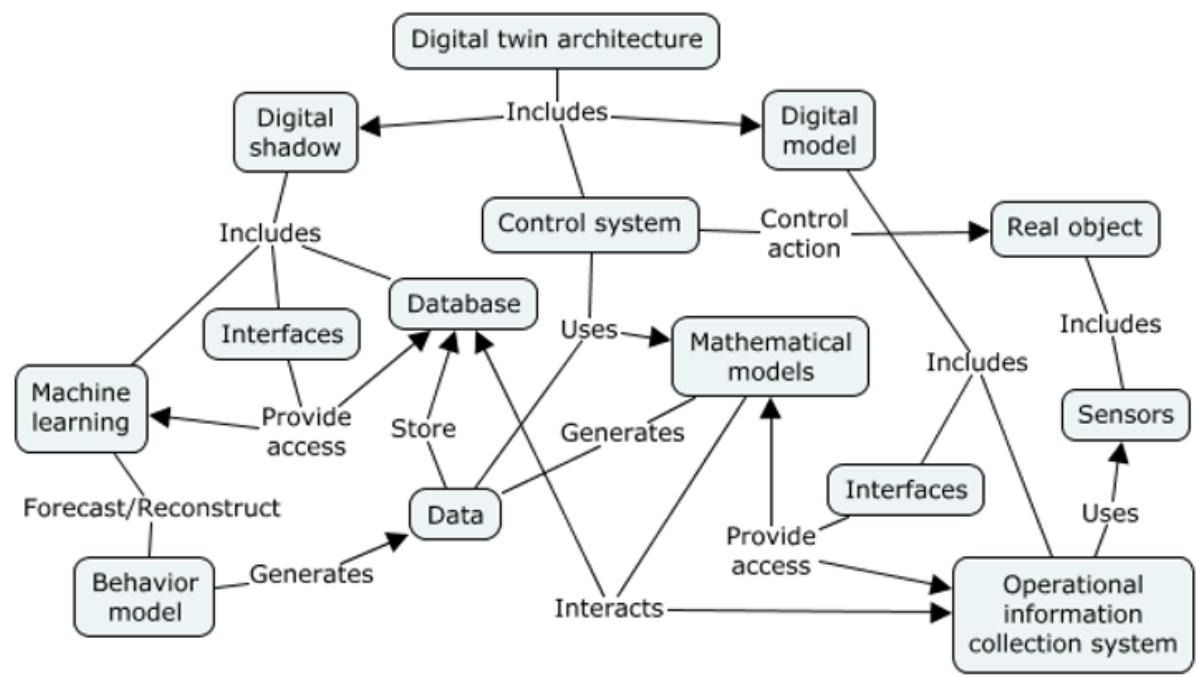

Fig. 7. Digital twin architecture 


\section{Further development}

For the possibility of using the developed digital twin on real objects, it is necessary to make the following improvements:

- Refine the architecture of the digital twin by dividing it into a digital shadow and a digital model:

The transformation will lead to an improvement in the maintainability and portability of the code, since the module with basic calculations (digital model) and the module for working with data (digital shadow) will be separated;

- Prepare the digital twin to work with large amounts of data collected from a real object:

When working with real objects of the electric power industry, such a problem arises as large amounts of data coming from sensors that must be stored and processed.

To solve the problem of data storage, it is proposed: to increase the efficiency of queries, to reduce the number of repetitions in data storage to a minimum and to optimize the relationships between tables in the database; this would require migrating the datastore to a non-relational database. To solve the problem of data processing, it is proposed to use Big Data methods, which will allow processing data within an acceptable time frame.

After putting the digital twin into operation, it will be possible to verify the models (both neural networks and mathematical ones) according to data obtained from a real object.

\section{Conclusion}

It is proposed to use an ontological approach when designing a digital twin and a database for a solar power plant. Ontological engineering is considered as a necessary stage in the construction of a digital twin. The use of ontological models to describe the structure of the database, its main components and relationships is shown. The main purpose of ontologies in the development of digital twins is the formal description and integration of all components: models, databases, knowledge bases (ontologies) and their relationships, and can be used to build digital twins of a solar power plant. The article also notes the subsequent directions of the development of the system, which are actively being worked out by the authors.

The results were obtained within the framework of the basic project of the ISEM SB RAS AAAA-A21121012090007-7 under the state assignment FWEU-20210007, with partial financial support from the RFBR grants No. 19-07-00351, 19-57-04003, 20-07-00994.

\section{References}

1. Order of the Government of the Russian Federation of June 9, 2020 No.1523-r "On approval of the Energy Strategy of the Russian Federation for the period up to $2035 "$

2. R. Rosen, G. Wichert, G. Lo, K. Bettengausen, IFACPapers OnLine, 567 (2015)

3. P.V. Semenov, R.P. Semishkur, I.A. Diachenko, Gas industry, No.7, 24 (2019)

4. M.N. Kramm, O.E. Bezborodova, O.N. Bodin, A.V. Svetlov, Measurement. Monitoring. Management. Control, No.1, 73 (2021)

5. D.A. Lysenko, Caspian Engineering and Construction Bulletin, No.4, 139 (2020)

6. R.G. Oganyan, Proceedings of higher educational institutions. North Caucasian region. Technical science, No.3, 34 (2020)

7. A.E. Saddik, IEEE MultiMedia, 25(No.2), 87 (2018)

8. D.S. Kokorev, Colloquium-journal. Technical science, No.10 (34), $3135 \quad$ (2019), $\quad$ DOI: 10.24411/2520-6990-2019-10264

9. A. Borovkov, Digital twins and digital shadows in the high-tech industry [Electronic resource]. URL: https://4science.ru/articles/Cifrovie-dvoiniki-icifrovie-teni-v-visokotehnologichnoipromishlennosti

10. S.K. Andryushkevich, Digital substation, No.12, 38 (2019)

11. Y. Shmotin, Digital Twin in Manufacturing: Tasks, Issues, Prospects [Electronic resource]. URL: http://www.uppro.ru/library/information_systems/project/d7fb9dd5 9e1ffa29.html.

12. A.V. Bukhanovsky, Energy and Industry of Russia, No.7 (363), (2019)

13. S.P. Kovalev, Systems and means of informatics, $\mathbf{3 0}$ (No.1), 66 (2020)

14. R. Studer, V. R. Benjamins, D. Fensel, Data \& Knowledge Engineering, 25, 161 (1998)

15. T. Gavrilova, D. Laird, In Industrial Applications of Semantic Web (Eds. Bramer M. and Terzyan V.), Springer, 61 (2005)

16. D.N. Karamov, I.V. Naumov, Power stations energoprogress, No.6, 21 (2020) 